$\xi=$

\title{
Complicated pantaloon umbilical hernia
}

\author{
Maurice E Asuquo ${ }^{1 *}$, Samuel Akpan ${ }^{2}$, Adams D Marwa ${ }^{2}$, Victor I Nwagbara ${ }^{1}$, John Ashindoitiang ${ }^{2}$ \\ ${ }^{1}$ Department of Surgery, University of Calabar, Calabar, Nigeria \\ ${ }^{2}$ Department of Surgery, University of Calabar Teaching Hospital, Calabar, Nigeria \\ *Corresponding authorE-mail: mauefas@yahoo.com
}

\begin{abstract}
Umbilical hernia is an abnormal protrusion of a whole viscus or part through the umbilical ring. Paraumbilical hernia is commonly found in adults protrude above or below the umbilicus. The occurrence of both types concurrently is described as pantaloon or saddle bag umbilical hernia. Complicated pantaloon umbilical hernia is rare. Presented was a case of complicated pantaloon umbilical hernia in a 55 year old multiparous female. Preoperative diagnosis was difficult especially in the setting of complication. Clinicians are urged to consider pantaloon umbilical hernia as a differential diagnosis of umbilical swellings.
\end{abstract}

Keywords: Umbilical Hernia; Pantaloon; Obstruction; Strangulation.

\section{Introduction}

Hernia is regarded as an abnormal protrusion of the whole viscus or part into an abnormal position with its coverings (Asuquo et al 2011). A pantaloon (reminiscent of trousers representing two sacs), dual (double sac), saddle bag (simulating a pair of covered pouches) hernia describes an ipsilateral direct and indirect hernia in the inguinal region occurring concurrently (Venkatesh,p1 ). The true (direct) umbilical hernia found commonly in neonates, and infants are a protrusion from the umbilical ring (Schumpelick \& Fitzgibbons 2007). The paraumbilical (indirect umbilical) hernias protrude above and or below the umbilicus and constitute the common type in adults (Taylor \& Kate 2015). The concurrent occurrence of the direct and indirect types is described as pantaloon umbilical hernia. Umbilical hernias constitute $10 \%$ of abdominal wall hernias. This may arise from weak fascia above the level with the umbilicus from multiple pregnancies, obesity, ascites and huge abdominal tumours (Taylor \& Kate 2015). Typically, a swelling is observed around the umbilicus with pain as the common reason for consulting the physician (Kulacoglu 2015). Clinical presentation ranges from reducible swelling with positive cough impulse to a painful, irreducible or obstructed/strangulated hernia, which should be treated with early surgery or as emergency respectively. The reason for the latter is that the neck of the umbilical hernia is usually narrow compared to the herniated mass, hence the need for elective herniorrhaphy. In view of the anatomy, the umbilicus is not a naturally clean part of the body. The use of antiseptic solutions may not destroy all the bacteria (Kulacoglu 2015). We present this rare case, first to be reported in our setting, of pantaloon hernia in the umbilicus to highlight the need for clinicians to consider pantaloon umbilical hernia as a differential diagnosis of umbilical swellings.

\section{Case report}

A 55-year-oldhousewife was referred from a secondary health facility to our emergency unit having been seen earlier in a prima- ry health care service with complaints of painful umbilical swelling and vomiting of 5 days and 2 days respectively. She became aware of the umbilical swelling when she was 16 years old and from then the swelling was not of concern to her as it disappeared spontaneously on lying down. Initially of the size of pulp of her little finger but gradually increased in size to twice the size of her thumb. Five days prior to presentation she observed that the swelling became irreducible and painful. Pain was of sudden onset, colicky and non radiating with no relieving factor but aggravated by movement and feeding. A couple of days prior to presentation, she vomited thrice. Vomitus was without blood stain. She last opened bowel 5 days prior to presentation and had poor appetite. Multiparous ( 1 male, 5 females), did not admit to history of lifting heavy load, constipation and chronic cough.

Examination revealed a middle-aged female in painful distress, afebrile, not pale, anicteric, mildly dehydrated no pedal oedema and peripheral lymphadenopathy. Vital signs were, temperature $36.2 \mathrm{o}$ C, pulse 88 beats /min, respiration 22 cycles/min and blood pressure (BP) 130/80 mmHg. Abdominal examination revealed a slightly distended abdomen that moved with respiration, no visible peristalsis with a vaguely bi-lobed spherical, tender, irreducible umbilical swelling that measured about $12 \mathrm{~cm} \times 9 \mathrm{~cm}$. There was no expansile cough impulse, and mass did not transilluminate. Liver, spleen was not palpable and kidneys not ballotable. Bowel sounds were hyperactive. Digital rectal examination was unremarkable and chest examination was normal. A clinical diagnosis of obstructed umbilical hernia was made.

Investigation results showed a PCV of $42 \%$, WBC- $8.4 \times 10^{9} / 1$ with a normal differential. Random blood sugar $6.4 \mathrm{mmol} / \mathrm{l}$, urea and electrolytes (U/E), Na- 135, K- 3.4, Cl-100, HCO3- 23, Urea15.5, Creatinine $244.9 \mathrm{mmol} / \mathrm{l}$. Serology for human immunodeficiency virus was non-reactive and urinalysis normal. Abdominal ultrasound scan (USS) report suggested supra umbilical hernia with partial intestinal obstruction.

Following resuscitation, she was offered an emergency herniorrhaphy. Access was through a midline incision over the umbilical swelling extending from about $5 \mathrm{~cm}$ above and below the swelling. Operative findings were two hernia sacs (pantaloon) separated by a fibrous structure, the upper part of the umbilical ring, Figure 1. 


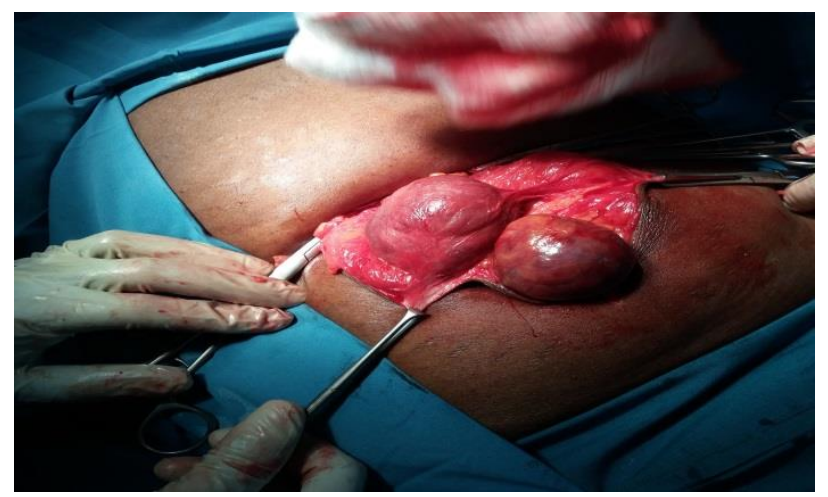

Fig. 1:Pantaloon Hernia, Top - Paraumbilical Hernia, Bottom - Umbilica Hernia.

The upper (paraumbilical) sac contained a gangrenous segment of the greater omentum with a hernia defect of about $3 \mathrm{~cm}$. The lower (umbilical) sac contained partially obstructed viable small bowel segment, with a defect of about $4 \mathrm{~cm}$, Figure 2.

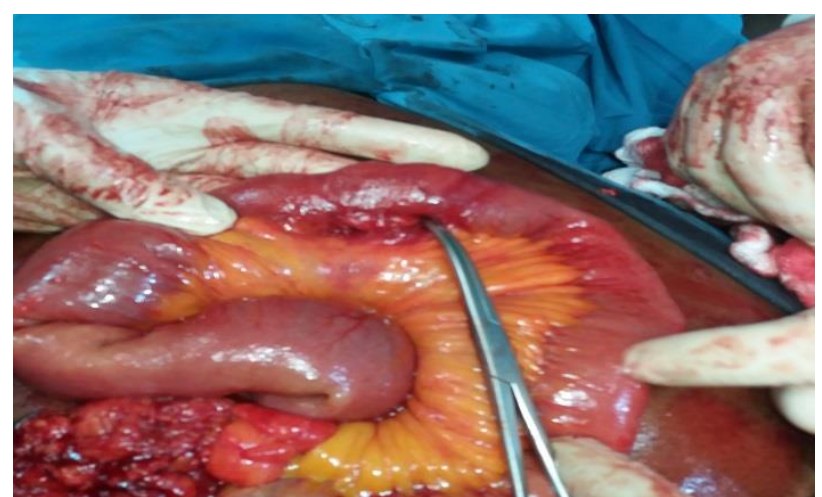

Fig. 2:Segment of Small Bowel Delivered from the Umbilical Hernia (Lower Sac, Fig. 1).

The gangrenous portion of the omentum was excised while viable small gut content of the lower sac was inspected and reduced. The edges of the fascia were freshened and repair done by mass closure with nylon 1 . Skin closure was with interrupted mattress suture with nylon 2/0. A post-operative diagnosis of pantaloon umbilical hernia was made.

Post operative period was uneventful until the $5^{\text {th }}$ day when the patient developed a fever $\left(38^{\circ} \mathrm{C}\right)$ and surgical site infection (SSI) in the upper third of the wound. Management included, wound swab for microscopy, culture and sensitivity $(\mathrm{m} / \mathrm{c} / \mathrm{s})$, removal of some sutures in the upper extremity of the wound and dressing initially with magnesium sulphate and subsequently honey. Antibiotic therapy initially ceftriazone and metronidazole, the former later changed to levofloxacin based on sensitivity. The wound healed by secondary intension and patient discharged $14^{\text {th }}$ post operative day. Follow up at the surgical outpatient department (SOPD) revealed satisfactory post operative state with good wound healing as at 30 days.

\section{Discussion}

The connection between the foetus and the placenta is marked by a scar known as the umbilicus. Its position notably varies located in the linea-alba based on patients' habitus (Ahmad 2011). The bulging of the abdominal content through a congenital or acquired weakness in the abdominal wall is described as hernia (Ahmad 2011, Nixon \&Tulloh 2013). A pantaloon or dual hernia depicts a double sac of a direct and indirect inguinal hernia of the same side with sacs found along both sides of the inferior epigastric vessels (Venkateshp1). Presented is a pantaloon hernia in an umbilical setting, Figure 1. The authors highlight the upper sac (paraumbilical) or indirect component from a defect in the linea alba and the lower umbilical sac as the direct component from the umbilical ring. It is further highlighted that the structure that represents the position of the inferior epigastric vessels in the pantaloon umbilical hernia is the fibrous tissue of the upper part of the umbilical ring.

The report of the relative occurrence of umbilical hernias as part of abdominal wall hernias varies; it ranged from 4-10\%: Taylor et al $10 \%$ (4), Kulacoglu 4-10\% (umbilical and epigastric) (Kulacoglu 2015) and Surg Wiki >4\% (Surg Wiki 2012). Umbilical hernias are generally reported to be more common in women though some report the contrary (Ahmad 2011). Comparative reports on the sex distribution show that umbilical and femoral hernias have female preponderance: umbilical (M(\%) 1, F(\%) 15), femoral $(\mathrm{M}(\%)$ 2, $\mathrm{F}(\%)$ 39) while inguinal had a male preponderance (M(\%) 96, F(\%) 45) (Surg Wiki 2012).

Bordering the umbilical canal are the linea alba in the anterior, umbilical fascia is a posterior while the medial edges of both rectus sheaths form the medial edges (Muysoms et al 2009). The later is important for the repair of the umbilical hernia which protrudes through the umbilical canal or ring. In the adult, the paraumbilical hernia is an acquired condition and distinct from umbilical hernia. It protrudes through a weak place in the linea alba immediately next to the true umbilicus commonly above as in this report, (Figure 1, upper hernia), again the rectus sheaths form the medial borders an important structure in the integrity of the repair in mass closure (Nixon \&Tulloh 2013, Surg Wiki 2012, Muysoms et al 2009).

Conditions which stretch the linea alba (median raphe) as multiple pregnancy, obesities, cirrhosis of the liver predisposes to opening of the defects (umbilical and paraumbilical) (Nixon \&Tulloh 2013). However, the umbilical hernia in the infant results from failure of the closure of the umbilical ring (Taylor \& Kate 2015). These hernias are usually typified by the narrow neck with omentum and small bowel as the usual contents in the sac, thereby increasing the risk of incarceration and strangulation (Taylor \& Kate 2015) as reported

In the definition of primary abdominal wall hernias, the European Hernia Society regards all hernias $3 \mathrm{~cm}$ above and below the umbilicus as umbilical hernia (Kulacoglu et al 2012). A recently simplified system of hernia classification as proposed by the European Hernia Society: 1), Primary or recurrent (P or R), 2), lateral, medial or femoral (L M F), 3). Defect size in fingers breaths estimated to be $1.5 \mathrm{~cm}$. We suggest that criteria no. 2 could be adapted to the umbilical setting with the following modification, above (A), below (B), right side (RS), and left side (LS) of the umbilicus for the paraumbilical hernia (PU) while the umbilical (U). Thus the presented pantaloon umbilical hernia will be classified as (1) P, PUA. 2, (2) P. U. 2.7

Clinical presentation may occur in the elective or emergency settings. In the earlier, the symptom is that of a lump around the umbilicus usually more prominent on standing and may disappear on lying down. In the later, presentation may be painful irreducible lump, obstruction and nausea and vomiting. The most common content of paraumbilical hernia is omentum as was our experience in addition to incarcerated or potentially ischemic bowel (Ahmad 2011). Notable complications at presentation are irreducibility, obstruction, strangulation, skin ulceration and rupture of hernia (Venkatesh, p2, Taylor \& Kate 2015).

In the adults, umbilical hernias should be electively repaired on account of the high risk of complications (incarceration and strangulation) compared to inguinal hernia (Taylor \& Kate 2015, Kulacoglu et al 2012). In the past 25 years in UK, umbilical and paraumbilical hernia repairs, including other abdominal wall hernias repairs increased from 5 to $14 \%$. Reports from Turkey portray a similar experience (Kulacoglu 2015). Herniorrhaphy is suitable for defects $<3 \mathrm{~cm}$, and the popular primary repair is Mayos "vestover-pants" repair. However, hernioplasty (mesh repair) has been associated with disease recurrence (Ahmad 2011). Our patient presented as emergency with a pantaloon hernia and was offered herniorrhaphy (suture repair), mass closure by approximation of the medial edges of the rectus sheaths in the midline. 
Complication following operation could be skin infection/mesh infection, hematoma, complications associated with bowel resection and recurrence. Due to the natural anatomical structure of the umbilicus, it is not a clean anatomical part even with the use of modern antiseptics. In view of the aforementioned, surgical site infection can be more frequent following repair when compared to the inguinal hernia (Museums et al 2009). It is not therefore, surprising with the report of $10 \%$ SSI even with the routine use of prophylactic antibiotic. Other factors that influence recurrence include large seromas, obesity and ascites (Kulacoglu et al 2012).

\section{Conclusion}

In conclusion, we report a case of complicated pantaloon umbilical hernia in an emergency setting. Clinicians are urged to be aware of this clinical entity as differential diagnosis of umbilical swellings and encourage patients to have elective surgery in view of the high risk of complications.

\section{References}

[1] Asuquo ME, Nwagbara VIC, Ifere MO. Epigastric hernia presenting as a giant abdominal interpariatal hernia. Int J Surg Case Reports 2011; 2: 243-245.https://doi.org/10.1016/j.ijscr.2011.07.008.

[2] Venkatesh M. Pantaloon hernia. Available at https://radiopaedia.org/articles/pantaloon-hernia, p1.

[3] Schumpelick V, Fitzgibbons RJ. Recurrent hernia, Prevention and treatment. Springer.Verlag Berlin. Heidelberg; 2007: 359-364.

[4] Taylor D, Kate V. Umbilical hernia. Repair. Available at http:// emedicine.medscape.com/article/2000990-overwiew. Updated October 19, 2015

[5] Kulacoglu H. Current options in umbilical hernia repair in adult patients. Ulus CerraliDerg 2015; 31: 157 161.https://doi.org/10.5152/UCD.2015.2955.

[6] Ahmad MF. Adult Umbilical and Paraumbilical hernia repair Available at http://www-surgeons.org.uk/General surgeryoperation-how to/adult umbilical and paraumbilical, 2011.

[7] Nixon SJ, Tulloh B. Abdominal wall hernia and umbilicus. In Williams NS, Bulsrode CJK, O'Connell PR editors. Bailey and Love's Short Practice of Surgery. $26^{\text {th }}$ Ed (International students ed) United States, Boca Raton, Florida. CRC Press (Taylor and Francis Group) 2013, p948-969

[8] Surg Wiki, Hernias. Available at http://www.surgi wiki.com/wiki/Hernias

[9] Muysoms FE, Miserez M, Berrevoet F, Campanelli G, Champault GG, Chalala E et al. Classification of primary and incisional abdominal wall hernia. Hernia 2009; 13: 407 414.https://doi.org/10.1007/s10029-009-0518-x.

[10] Kulacoglu H, Yazicioglu D, Ozyaylali I. Prosthetic repair of umbilical hernias in adults with local Anaesthetic in a day case setting a comprehensive report from a specialized hernia centre. Hernia 2012; 16: 163-170.https://doi.org/10.1007/s10029-011-0888-8. 